\title{
Thermal, trapped and chromo-natural inflation in light of the swampland criteria and the trans-Planckian censorship conjecture
}

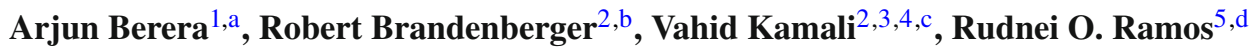 \\ ${ }^{1}$ School of Physics and Astronomy, University of Edinburgh, Edinburgh EH9 3FD, UK \\ ${ }^{2}$ Department of Physics, McGill University, Montreal, QC H3A 2T8, Canada \\ ${ }^{3}$ Department of Physics, Bu-Ali Sina (Avicenna) University, Hamedan 65178-016016, Iran \\ ${ }^{4}$ School of Physics, Insitute for Research in Fundamental Sciences (IPM), Tehran 19538-33511, Iran \\ ${ }^{5}$ Departamento de Física Teórica, Universidade do Estado do Rio de Janeiro, 20550-013 Rio de Janeiro, RJ, Brazil
}

Received: 26 June 2020 / Accepted: 15 May 2021 / Published online: 25 May 2021

(C) The Author(s) 2021

\begin{abstract}
We consider thermal, trapped and chromo-natural inflation in light of the swampland criteria and the TransPlanckian Censorship Conjecture (TCC). Since thermal inflation occurs at energies low compared to those of Grand Unification, it is consistent with the TCC, and it is also consistent with the refined swampland conditions. Trapped and chromo-natural inflation are candidates for primordial (high energy scale) inflation. Since in both of these scenarios there are effective damping terms in the scalar field equation of motion, the models can easily be consistent with the swampland criteria. The TCC, on the other hand, constrains these scenarios to only take place at low energies.
\end{abstract}

\section{Introduction}

The inflationary scenario [1-10] has become the paradigm of early universe cosmology. Not only does it explain the near homogeneity, isotropy and spatial flatness of the observed universe, but it also provides a causal mechanism [11,12] for the origin of structure. Inflation is generally described at the level of an effective field theory in which the almost constant potential energy density of a scalar field leads to almost exponential expansion of space. It is easy to show [13] that with an appropriately chosen scalar field potential it is possible to obtain whatever background cosmologyone wants.

\footnotetext{
a e-mail: ab@ph.ed.ac.uk

be-mail: rhb@physics.mcgill.ca (corresponding author)

c e-mail: vkamali@ipm.ir

de-mail: rudnei@uerj.br
}

In light of this unsatisfactory situation, it is of great interest to ask which effective scalar field models in fact can be consistent with fundamental physics, in particular superstring theory. There has recently been an active research program to address this issue, an effort which goes under the name swampland program (see e.g. [15-17] for reviews). Note that the swampland program may also impose constraints on cosmological models alternative to inflation which are based on effective point particle field theories. On the other hand, there are alternatives to inflation (e.g. String Gas Cosmology [14]) which are based on fundamental principles of superstring theory, do not make use of effective scalar fields, and are hence unconstrainted by the swampland constraints.

Specifically, it has been suggested that the potential of a canonically normalized scalar field (in particular of the scalar field responsible for generating the inflationary expansion) must satisfy a number of conditions (the so-called swampland criteria) in order to allow an embedding in superstring theory (see [18,19] for original articles and [15-17] for reviews). On the other hand, the Trans-Planckian Censorship Conjecture (TCC) [20] is a proposed constraint on cosmological models coming from demanding that physics on scales larger than the cosmological horizon be shielded from the non-unitarity [21] of the effective field theory description of the cosmological model, and can be viewed as a generalization of Penrose's Cosmic Censorship Conjecture [22,23] to the case of cosmology (see e.g. [24] for a discussion of these aspects). The swampland criteria rule out single field slow-roll inflation models and false vacuum inflation. They also rule out a model in which Dark Energy is a bare cosmological constant, while remaining consistent (given the current status of observations) with quintessence models of Dark Energy $[25,26]$. The TCC puts a tight constraint on slow-roll inflationary models [27], forcing the energy scale of such a period of inflation 
to be smaller than about $10^{10} \mathrm{GeV}$, thus yielding a negligibly small amplitude of gravitational waves. ${ }^{1}$

The swampland criteria, however, do not rule out all inflationary models. Some multi-field models may remain consistent [41], and the warm inflation scenario [42] can easily be consistent with the criteria [43-46]. In this paper we consider representative examples of three interesting classes of non-slow-roll inflation models and study what kind of constraints the swampland criteria and the TCC place on them. The models we consider are thermal inflation [47], trapped inflation [48] and chromo-natural inflation [49]. Trapped and chromo-natural inflation follow the basic warm inflation ideas of particle production during inflation and the associated additional sources of friction in the dynamics of the inflaton, which just as for warm inflation therefore allow steep inflaton potentials. Given the success of warm inflation in addressing the swampland criteria [43-46], it motivates us to therefore study these other models. Particle production during inflation not only implies a modified inflationary dynamics, it also implies the possibility of a thermal bath. This property also is present in thermal inflation, although in that scenario it is present simply as an initial condition and there is no particle production mechanism to sustain it. Thus in thermal inflation the presence of the thermal state is short lived and is not present long enough to affect large scale observable perturbations. Nevertheless there are some similarities again with warm inflation that motivate a closer look. The effects of a radiation bath and the reheating problem have also been discussed in the context of the swampland criteria [50]. Since both trapped and thermal inflation have some similarities to the reheating problem, this gives an additional motivation for studying these models here. We show that all three models above to varying degree can be made consistent with the swampland criteria. We also find that thermal inflation is clearly consistent with the TCC. For trapped inflation and chromo-natural inflation, the TCC leads to similar constraints as it does for standard slow-roll inflation.

The structure of this note is as follows. In the following section we briefly review the swampland criteria and the TCC. Then follow three sections in which we one by one discuss thermal inflation, trapped inflation and chromo-natural inflation. For each of these models, we first mention their motivation, and then study the constraints. We are working in the context of a standard Friedmann-Robertson-WalkerLemaitre cosmology with the space-time metric given by the line element

$d s^{2}=d t^{2}-a(t)^{2} d \mathbf{x}^{2}$

\footnotetext{
1 The constraint becomes even stronger if the effects of a preinflationary radiation phase are taken into account $[28,29]$, but weakens in the case of power law inflation [30-34], or if non-standard cosmology after reheating is allowed [30-33].
}

where $t$ is physical time, $\mathbf{x}$ are the comoving spatial coordinates and $a(t)$ is the scale factor. The expansion rate is $H(t) \equiv \dot{a} / a$, the dot indicating a derivative with respect to time. We work in natural units in which Planck's constant, the speed of light and Boltzmann's constant are set to 1 . The reduced Planck mass is denoted by $M_{\mathrm{Pl}}$.

\section{Swampland Criteria and Trans-Planckian Censhorship}

Cosmological inflation [1-10] is usually studied in the context of effective field theory when a scalar matter field $\phi$ is coupled to Einstein gravity. Effective field theory is very successful when studying low energy phenomena, but it is incomplete in the high energy limit, and it is this high energy limit which is important for early universe cosmology. Superstring theory is our best candidate for a theory which unifies matter and gravity at high energies. Thus, it is of great importance to ask which models of effective scalar fields are consistent with superstring theory (these are said to lie in the landscape) and which are not (they are in the swampland). There are a number of criteria which have been proposed (see [15-17] for reviews). We will focus on two of these criteria most important for inflationary cosmology, in the form discussed in [19].

The first suggested criterion [18] for an effective field theory consistent with string theory is that the range of field values $\Delta \phi$ which the dynamics explores is smaller than the Planck scale,

$\Delta \phi<c_{1} M_{\mathrm{Pl}}$,

where $c_{1}$ is a constant of the order one. The argument in support of this criterion comes from the fact the scalar fields in a low energy effective scalar field model originate from moduli of a string theory compactification, e.g. the radius of an internal cycle. As the modulus changes, the masses of string states such as string winding modes or Kaluza-Klein modes change. In particular, if such a modulus field moves by more than $c_{1} M_{P l}$, then towers of string states become low mass, signalling a breakdown of an effective field theory description. The second criterion [19] concerns the slope of the scalar field potential $V(\phi)$. For scalar fields coming from string theory, the slope has to be sufficiently large,

$\frac{V^{\prime}}{V} M_{\mathrm{Pl}}>c_{2}$

where $c_{2}$ is another constant of the order one, and a prime denotes a derivative with respect to $\phi$. The origin of this condition is the following: the potential for a modulus field typically arises from some stringy mechanism which stabi- 
lizes the modulus field. In examples [19], this potential has a slope given by the string scale (see e.g. [51,52] for examples). This condition applies to a rolling scalar field which dominates the energy density of the universe (see e.g. [53] for a derivation from entropy considerations). For fields located near a local maximum of their potential, it is possible than Eq. (3) is not satisfied, as long as the potential is sufficiently tachyonic $[53,54]$ :

$\frac{V^{\prime \prime}}{V} M_{\mathrm{Pl}}^{2}<-c_{3}$,

where $c_{3}$ is once again a constant of the order one.

Note that there is still a lot of disagreement about the validity of the swampland constraints. In fact, a large number of string-motivated models of inflation have been proposed (see e.g. the textbook treatment in [55]). However, as again mentioned in [55], none of the proposed constructions are under full control, and in fact no-go theorems for a de Sitter phase have been suggested [56-60].

The condition (3) clearly is in conflict with simple single slow-roll scalar field models with canonically normalized kinetic terms, since the left-hand side of Eq. (3) is the first slow-roll parameter which is supposed to be much smaller than one. The condition (2) excludes most large field models of inflation with canonical kinetic terms ${ }^{2}$. This is also problematic since it is large field models of inflation in which the slow-roll trajectory is a local attractor in initial condition space [62] (see also [63] for a review), whereas this attractor nature is not present in small field models [64].

A different constraint on cosmological models comes from the recently postulated Trans-Planckian Censorship Conjecture (TCC) [20]. According to this conjecture, transPlanckian modes (modes with constant comoving wavelength) must remain hidden by cosmological horizons. If we consider the mode corresponding to the Planck length $l_{p l}$ at some initial time $t_{i}$, then its physical wavelength must remain smaller than the Hubble horizon $H^{-1}\left(t_{R}\right)$ at all later times $t_{R}$, i.e.,

$l_{p l} \frac{a\left(t_{R}\right)}{a\left(t_{i}\right)}<H^{-1}\left(t_{R}\right)$

or equivalently

$\ln \left(\frac{M_{\mathrm{Pl}}}{H}\right)>N$

where $N$ is the number of e-folds of inflation. This condition imposes an upper bound on the duration of inflation. It has

\footnotetext{
2 Note [61] for a study of models with non-canonical kinetic terms for which inflation arises at very large field values.
}

been argued that an $\mathcal{O}(1)$ factor can appear on the left hand side of the last expression [65].

If inflation is to provide a causal mechanism for producing all of the structures we observe today, the comoving scale corresponding to the current Hubble radius today (time $t_{0}$ ) must originate inside the Hubble horizon at the beginning of inflation (time $t_{i}$ ), i.e.,

$H\left(t_{0}\right)^{-1} \frac{a\left(t_{i}\right)}{a\left(t_{R}\right)} \frac{a\left(t_{R}\right)}{a\left(t_{0}\right)}<H\left(t_{i}\right)^{-1}$,

where here $t_{R}$ corresponds to the end of inflation. The above condition imposes a lower bound on the duration of inflation. The upper bound from Eq. (5) and the lower bound from Eq. (7) on the duration of inflation are consistent provided that the energy scale $V_{0}^{1 / 4}$ of inflation is

$V_{0}^{1 / 4}<10^{10} \mathrm{GeV}$

which leads to an upper bound on the tensor to scalar ratio $r$ of

$r<10^{-30}$

assuming that there is a mechanism to generate scalar fluctuations of the observed magnitude. These last two equations were obtained assuming almost exponential inflation and are relaxed for models such as power-law inflation [34]. However the $\mathcal{O}(1)$ correction argued by the modified TCC could increase the upper bound on the energy scale to accommodate even up to the GUT scale, which is most typically associated with inflation.

Note that the TCC - at least in the way presented above - is a condition on the applicability of an effective field theory treatment of an inflationary phase. In a non-perturbative approach to inflation like e.g. in [35-40], the argument does not apply, as pointed out in [66]. ${ }^{3}$

\section{Thermal Inflation and the Swampland}

Thermal inflation is a phase of late time inflation which was proposed in Ref. [47] as a way to dilute unwanted moduli fields which are produced in the early universe, in particular in supersymmetric models. Since it is a low scale model of inflation, and there is no requirement that the comoving scale corresponding to the current Hubble radius emerges from inside the Hubble radius during the period of thermal inflation (in fact, we do not want this to be the case), it is natural to

\footnotetext{
3 The criticism of the TCC raised in [67], on the other hand, in our opinion misses the key role which the expansion of the background space implies for the applicability of an effective field theory in light of the existence of an ultraviolet cutoff.
} 
expect the thermal inflation scenario to be consistent with the TCC. Here we wish to explore whether it can be consistent with the swampland criteria.

Thermal inflation postulates a new complex scalar field $\phi$ with a potential of the form

$V=V_{0}-m_{0}^{2}|\phi|^{2}+\sum_{n=1}^{\infty} \lambda_{n} M_{\mathrm{Pl}}^{-2 n}|\phi|^{2 n+4}$,

where the quadratic term comes from soft supersymmetry breaking, with $m_{0}$ being close to the scale of electroweak symmetry breaking. $m_{0}$ is taken to be in the range between $10^{2}$ and $10^{3} \mathrm{GeV}$. The higher order terms are nonrenormalizable ones with coupling constants $\lambda_{n} \sim 1$ for the theory to be valid up to the Planck scale $M_{\mathrm{Pl}}$. Note the absence of a quartic renormalizable term in the potential. This is justified if we assume that $\phi$ is a flat direction before supersymmetry breaking at the perturbative level.

If the n'th term in Eq. (10) dominates in the sum, we have a nonvanishing vacuum expectation value for $\phi$ given by $|\phi|=M$, where

$M^{2 n+2} M_{\mathrm{Pl}}^{-2 n}=2(n+1)\left[2(n+1)(n+2) \lambda_{n}\right]^{-1} m_{0}^{2}$.

For $n=1$ (the case we will focus on in the following) we find that $M$ is in the range between $10^{10}$ and $10^{11} \mathrm{GeV}$. By requiring that $V(M)=0$ we have that the constant term $V_{0}$ in Eq. (10) is given by

$V_{0}=2(n+1)[2(n+2)]^{-1} m_{0}^{2} M^{2}$.

From the form of the potential we then obtain $V^{\prime \prime}(M) \ll$ $M^{2}$, which thus yields a very flat potential, and the field $\phi$ is hence called a "flaton".

If we assume that $\phi$ is coupled to other fields $\chi$ which are in thermal equilibrium at a temperature $T$, and the masses of these field are smaller than $T$, then the potential (10) obtains finite temperature corrections $\delta_{T} V$ of the form [68]

$\delta_{T} V=\frac{1}{12} g^{2} T^{2}|\phi|^{2}$,

where we have taken a standard coupling between $\phi$ and $\chi$ of the form $g^{2}|\phi|^{2} \chi^{2}$. Hence, the flaton field will be trapped near the origin for $T>T_{c}$, where

$T_{c}=2 \sqrt{3} g^{-1} m_{0}$.

Thermal inflation begins when the potential energy of the $\phi$ field (trapped at the origin in field space) starts to dominate over the energy density in the thermal background. This occurs at a temperature $T_{I}$ given by

$T_{I}=\left(g^{*}\right)^{-1 / 4}\left(\frac{2}{3}\right)^{1 / 4} m_{0}^{1 / 2} M^{1 / 2}$,

where $g^{*}$ is the number of degrees of freedom in the thermal bath, and where we have taken the case $n=1$ for simplicity. The number $N$ of e-foldings of thermal inflation is then determined by

$e^{N}=\frac{T_{I}}{T_{c}}$

which yields

$N=\frac{1}{2} \ln \left(\frac{M}{m_{0}}\right)+\ln \tilde{g}$,

where

$\tilde{g} \equiv 6^{-3 / 4}\left(g^{*}\right)^{-1 / 4} g$.

Let us now study the compatibility between thermal inflation and the TCC. In the spirit of strengthening the TCC bound $[28,29]$ we assume that the universe is dominated by radiation back to the Planck time. The TCC criterion then demands that the comoving scale corresponding to the Planck length at the Planck time remains smaller than the Hubble radius at the end of the period of thermal inflation, i.e., at the time $t_{c}$ we have that

$l_{p l} \frac{a\left(t_{c}\right)}{a\left(t_{I}\right)} \frac{a\left(t_{I}\right)}{a\left(t_{p l}\right)}<t_{c}^{-1}$.

Making use of our assumption of pre-inflationary radiation domination, the first ratio on the left-hand side of Eq. (19) equals $T_{p l} / T_{I}$ (where $T_{p l}$ is the Planck temperature), while the second ratio is $e^{N}$. Parametrizing the value of $m_{0}$ as

$m_{0} \equiv 10^{2+\eta} \mathrm{GeV}$,

in terms of a constant $\eta$, the condition (19) becomes (dropping constants of order one, and setting $g^{*}=1$ )

$g<10^{12-\eta / 2}$

As expected, we find that the TCC can almost trivially be satisfied.

We now turn to the swampland constraints. Since $M \ll$ $M_{\mathrm{Pl}}$ and the field $\phi$ evolves from $\phi=0$ to $|\phi|=M$, the field range condition (2) is also trivially satisfied. The de Sitter conditions (3) and (4) are less trivial. Since the potential is very flat, there could be important constraints. Note that 
it is the potential without temperature corrections which is relevant for the de Sitter condition. Indeed, since

$$
\frac{\left|V^{\prime}\right|}{V} \simeq \frac{2 m_{0}^{2}|\phi|}{V_{0}}
$$

and since $|\phi|$ is close to the origin, the condition (3) is grossly violated. To be more specific, we can evaluate the condition at the field value $\left|\phi_{H}\right|$ given by its quantum expectation value during inflation, i.e.,

$\left|\phi_{H}\right| \sim H\left(T_{I}\right)$

Modulo numerical factors the condition (3) becomes

$e^{-4 N} g^{2}>1$

which is extremely hard to realize. However, since during inflation $\phi$ is trapped at the field origin, there is another way in which the model can be consistent with the (refined) swampland constraints. This is the case if Eq. (4) is satisfied. Now, since the potential is tachyonic at the origin in field space and

$\frac{\left|V^{\prime \prime}\right|}{V} M_{\mathrm{Pl}}^{2}=3\left(\frac{M_{\mathrm{Pl}}}{M}\right)^{2}$

(again for the case $n=1)$. Since $M \ll M_{\mathrm{Pl}}$ we see that Eq. (4) is trivially satisfied.

Thus, we have shown that thermal inflation is consistent both with the TCC and with the refined swampland conditions.

\section{Trapped Inflation and the Swampland}

Trapped inflation is based on basic warm inflation ideas of particle production during inflation and the corresponding damping effects on the inflaton evolution. The scenario obtains inflation on steep potentials which is motivated by string theory constructions [48]. In the context of string theory, scalar fields which arise in the low energy effective field theory of our four space-time dimensional world are moduli fields of the string theory, e.g., size and shape moduli associated with the compact extra-dimensional space. It is known that for such moduli there are enhanced symmetry values at which towers of string states which are of the string scale in Minkowski space-time become low mass (mass smaller than the typical energy scale of the modulus field dynamics). If the modulus field is rolling, then these string states can be parametrically produced when the field passes through such an enhanced symmetry point [69-71]. This process is analogous to the parametric production of particles at the end of inflation when the inflaton field oscillates about the minimum of its potential [72-77] (see, e.g., [78,79] for reviews). The produced particles contribute to the effective potential of the modulus field and tend to slow it down.

We expect that the swampland criteria may be satisfied in the trapped inflation scenario, and in the following we will verify that this is indeed the case. The constraints on the TCC, on the other hand, are identical to the ones for standard slow-roll inflation.

The Lagrangian density which describes the interaction of the inflaton modulus field $\phi$ with the other fields $\chi_{i}$ is the same as the distributed mass model of warm inflation [80,81],

$\mathcal{L}_{I}=\frac{1}{2} g^{2} \sum_{i}\left(\phi-\phi_{i}\right)^{2} \chi_{i}^{2}$

where the $\chi_{i}$ represent particles which become light (with mass less than the Hubble parameter) at the enhanced symmetry points $\phi=\phi_{i}$, and $g$ is the coupling constant (which is taken to be independent of $i$ ). The $\chi_{i}$ fields are taken to have canonical kinetic terms.

As studied in Ref. [70], $\chi_{i}$ particles are resonantly produced as $\phi$ crosses the value $\phi_{i}$. The number density of $\chi_{i}$ particles is

$n_{\chi_{i}}(t) \simeq \frac{g^{3 / 2}}{(2 \pi)^{3}} \dot{\phi}^{3 / 2}\left(t_{i}\right) \frac{a^{3}\left(t_{i}\right)}{a^{3}(t)}$,

where the last factor comes from the redshifting of the number density of particles after they are produced at the time $t_{i}$.

The production of $\chi$ particles extracts energy from the $\phi$ field and hence leads to an equation of motion of the form [48]

$\ddot{\phi}+3 H \dot{\phi}+V^{\prime}(\phi)+\sum_{i} \frac{g^{5 / 2}}{(2 \pi)^{3}} \dot{\phi}^{3 / 2}\left(t_{i}\right) \frac{a^{3}\left(t_{i}\right)}{a^{3}(t)}=0$.

If the separation $\Delta$ between neighboring enhanced symmetry points is small (we take them to be equally spaced), then we can approximate the sum in the equation of motion (28) by an integral, and the integral is dominated at the final crossing point. Thus,

$\sum_{i} g\left|\phi-\phi_{i}\right| n_{\chi} \simeq \frac{g^{5 / 2}}{3 H(2 \pi)^{3}} \dot{\phi}^{5 / 2}$

In this way, the approximate form of the inflaton equation of motion becomes

$\ddot{\phi}+3 H \dot{\phi}+V^{\prime}(\phi)+\frac{g^{5 / 2}}{3 H \Delta(2 \pi)^{3}} \dot{\phi}^{5 / 2}=0$. 
Trapped inflation arises if

$|\ddot{\phi}| \ll 3 H|\dot{\phi}| \ll\left|V^{\prime}\right|$.

In this case Eq. (30) yields the result

$\dot{\phi} \simeq-\frac{\left[3 H \Delta(2 \pi)^{3}\left|V^{\prime}\right|\right]^{2 / 5}}{g}$.

The first calculation we do here is to see whether the second part of the inequality in Eq. (31) is consistent with the de Sitter condition (3). Assuming that the potential energy density dominates, we can express $H$ in terms of $V$ and the Planck mass. The condition (31) becomes

$\frac{\left|V^{\prime}\right|}{V} M_{\mathrm{Pl}}>g^{-5 / 3} 3^{7 / 3}(2 \pi)^{2} V^{1 / 6} M_{\mathrm{Pl}}^{-4 / 3} \Delta^{2 / 3}$.

This is a lower bound on the relative slope of the potential and not an upper bound as one may have first expected. Thus, there is no inconsistency with the de Sitter condition.

Next, we need to verify that the slow-roll condition is consistent with the swampland criteria. The slow-roll parameter $\epsilon$ is given by [48]

$\epsilon=\frac{3\left(\dot{\phi}^{2}+\sum_{i} g\left|\phi-\phi_{i}\right| n_{\chi}\right)}{2 V}$.

We will study the two terms on the right-hand side of the above separately. First, making use of Eq. (32), we find that the condition $\dot{\phi}^{2} / V \ll 1$ becomes

$M_{\mathrm{Pl}} \frac{\left|V^{\prime}\right|}{V} \ll \frac{M_{\mathrm{Pl}}}{\Delta} \frac{M_{\mathrm{Pl}}}{V^{1 / 4}} \frac{1}{3(2 \pi)^{3}}$.

Since the energy scale $V^{1 / 4}$ of inflation is much lower than the Planck mass, and since $\Delta \ll M_{\mathrm{Pl}}$ we see that the right hand side of the above is much larger than one, and so the condition (35) can easily be consistent with the de Sitter constraint (3). Now, let us move on to the second term in Eq. (34). To study the condition

$\frac{\sum_{i} g\left|\phi-\phi_{i}\right| n_{\chi}}{2 V} \ll 1$,

we make use of Eq. (29) and obtain

$M_{\mathrm{Pl}} \frac{\left|V^{\prime}\right|}{V} \ll \frac{2 M_{\mathrm{Pl}}}{\Delta}$,

which once again can be consistent with the de Sitter criterion (3) provided that $\Delta \ll M_{\mathrm{Pl}}$.

We thus conclude that trapped inflation can be consistent with the swampland criteria. However, since trapped inflation involves almost exponential expansion, the TCC criterion applies without change and implies the upper bound (8) on the energy scale of inflation and the upper bound (9) on the tensor to scalar ratio. The analysis so far has not examined the parameter regime consistent with observation. Accounting for that makes it more difficult to remain consistent with the swampland criteria. For example, for the inflaton potential $m^{2} \phi^{2} / 2$, the region in the space of the two parameters of the model, $\left(g, m / M_{\mathrm{Pl}}\right)$, consistent with observational constraints on tilt, $r$, and non-Gaussianity as evaluated in Ref. [48] becomes very narrow when also accounting for the swampland criteria.

\section{Chromo-Natural Inflation and the Swampland}

Chromo-natural inflation [49] is a proposal to obtain inflation on a steep potential by coupling the inflaton field, which is assumed to be an axion field, via a Chern-Simons coupling to a non-Abelian gauge field. The proposed matter Lagrangian density is

$\mathcal{L}=\frac{1}{2} \partial_{\mu} \phi \partial^{\mu} \phi-\mu^{4}\left[1+\cos \left(\frac{\phi}{f}\right)\right]-\frac{\lambda}{8 f} \phi F_{\mu \nu}^{a} \tilde{F}_{a}^{\mu \nu}$

where $\tilde{F}$ is the dual of the field strength tensor $F, f$ is the analog of the axion decay constant, $\lambda$ is a dimensionless coupling constant, and $a$ is a group index.

In the absence of coupling to the gauge field, inflation would only be possible for $f>M_{\mathrm{Pl}}$ (this is the natural inflation [82] scenario), and this would lead to a conflict with both the distance criterion (2) and the de Sitter condition (3) or (4). However, the coupling to the gauge field can provide an effective friction analogous of what occurs for trapped inflation.

In order for the scenario to work, there must be a homogeneous gauge field configuration, and this is not possible for a $U$ (1) gauge field. For an $S U$ (2) gauge field (and similarly for any larger group which contains a $S U$ (2) subgroup), we can construct a homogeneous gauge field configuration

$A_{0}^{a}=0$

$A_{i}^{a}=a(t) \psi(t) \delta_{i}^{a}$,

where the index $i$ is the usual spatial index. ${ }^{4}$ The equation of motion for the axion field $\phi$ then becomes

$\ddot{\phi}+3 H \dot{\phi}-\frac{\mu^{4}}{f} \sin \left(\frac{\phi}{f}\right)=3 \tilde{g} \frac{\lambda}{f} \psi^{2}(\dot{\psi}+H \psi)$,

where $\tilde{g}$ is the gauge coupling constant.

It was shown [49] that natural initial conditions for $\psi$ lead to the possibility of obtaining inflation (driven by the potential energy of $\phi$ ) for values $f \ll M_{\mathrm{Pl}}$. In this case, it is

\footnotetext{
${ }^{4}$ This was first pointed out in [83-85] under the name gauge-flation.
} 
obvious that the swampland criteria are satisfied. Once again, however, the TCC imposes the upper bound (8) on the energy scale of inflation, and the bound (9) on the tensor to scalar ratio.

\section{Conclusions}

We have shown that the thermal inflation, trapped inflation and chromo-natural inflation (and more generally gaugeflation) scenarios all satisfy the swampland criteria. The latter two models both rely on damping effects on the inflaton due to interaction with other fields, following the same idea as in warm inflation, which is one general prototype for consistency with the swampland criteria [43-46].

Whereas thermal inflation is also consistent with the TCC (because it is by construction a low scale model with a short period of inflation), the TCC imposes the same upper bounds on the energy scale of inflation, and on the tensor to scalar ratio, as what is obtained for single field slow-roll inflation. However, the modified TCC could substantially relax this upper bound on the energy scale.

It is fair to recall, however, that there are controversies about the swampland conjectures within the string theory community. This concerns both the construction of explicit de Sitter string theory vacua, and of metastable inflationary trajectories (see [55] for a review of many proposed constructions, and [56-60,86] for discussions concerning difficulties in these constructions). Nonetheless, the swampland conjectures give a set of conditions that an effective field theory may have to satisfy in order to be consistent with string theory. It is, therefore, worth while to explore their consequences for different inflation models, like in the ones we have considered in this work.

We wish to close with a couple of other comments. Another point that has recently been reiterated [66] is that inflation models should be independent of the high energy quantum gravity theory. In the effective field theory framework, loop calculations are truncated at the high energy cutoff scale under the assumption that the high energy physics will properly take care of the renormalization. A perfect decoupling from the high energy physics is when the effective low energy theory is conventionally renormalizable, and thus contains operators of dimension four or less. For all other cases, the extent of decoupling in the theory is dependent on the type of questions being asked from the theory.

Ever since the single field monomial chaotic models have been ruled out by observation, it has been difficult to realize the cold single field inflation scenario with a conventionally renormalizable theory. In particular, single field models which are a best fit to observations [87], have nonrenormalizable operators that play an essential role in the inflaton dynamics. These nonrenormalizable operators are all rem- nants of the high energy quantum gravity theory. It is therefore quantum gravity that determines the form of the potential and thus all of the observational signatures. Since the basic questions asked from such theories are inherently based on the high energy physics, there is no decoupling in such theories. Models with scalar fields non-minimally coupled to gravity are still consistent with observation for $\phi^{4}$ inflaton potentials. However such theories are inherently dependent on classical gravity working, and so by extension would not be independent of quantum gravity effects either. In contrast to this, there are several warm inflation models that are conventionally renormalizable, going back to the earliest distributed mass model [80], the two-stage model [88], and the more recent Warm Little Inflation model [89].

Acknowledgements A. B. is supported by STFC. The research at McGill is supported in part by funds from NSERC and from the Canada Research Chair program. RB is grateful for hospitality of the Institute for Theoretical Physics and the Institute for Particle Physics and Astrophysics of the ETH Zurich during the completion of this project. R.O.R. is partially supported by research grants from Conselho Nacional de Desenvolvimento Científico e Tecnológico (CNPq), Grant No. 302545/2017-4, and Fundação Carlos Chagas Filho de Amparo à Pesquisa do Estado do Rio de Janeiro (FAPERJ), Grant No. E26/202.892/2017. VK would like to acknowledge the McGill University Physics Department for hospitality and the McGill Space Institute for partial financial support. He also thanks Cumrun Vafa and Shahin Sheikh-Jabbari for discussions.

Data Availability Statement This manuscript has no associated data or the data will not be deposited. [Authors' comment: Since this is a purely theoretical study, there is no associated data.]

Open Access This article is licensed under a Creative Commons Attribution 4.0 International License, which permits use, sharing, adaptation, distribution and reproduction in any medium or format, as long as you give appropriate credit to the original author(s) and the source, provide a link to the Creative Commons licence, and indicate if changes were made. The images or other third party material in this article are included in the article's Creative Commons licence, unless indicated otherwise in a credit line to the material. If material is not included in the article's Creative Commons licence and your intended use is not permitted by statutory regulation or exceeds the permitted use, you will need to obtain permission directly from the copyright holder. To view a copy of this licence, visit http://creativecomm ons.org/licenses/by/4.0/.

Funded by SCOAP ${ }^{3}$.

\section{References}

1. A.H. Guth, The inflationary universe: a possible solution to the horizon and flatness problems. Phys. Rev. D 23, 347 (1981)

2. A.H. Guth, The inflationary universe: a possible solution to the horizon and flatness problems. Adv. Ser. Astrophys. Cosmol. 3, 139 (1987). https://doi.org/10.1103/PhysRevD.23.347

3. R. Brout, F. Englert, E. Gunzig, The creation of the universe as a quantum phenomenon. Ann. Phys. 115, 78 (1978)

4. A.A. Starobinsky, A new type of isotropic cosmological models without singularity. Phys. Lett. B 91, 99 (1980) 
5. K. Sato, First order phase transition of a vacuum and expansion of the universe. Mon. Not. R. Astron. Soc. 195, 467 (1981)

6. L.Z. Fang, Entropy generation in the early universe by dissipative processes near the Higgs' phase transitions. Phys. Lett. B 95, 154 (1980). https://doi.org/10.1016/0370-2693(80)90421-9

7. A.D. Linde, A new inflationary universe scenario: a possible solution of the horizon, flatness, homogeneity, isotropy and primordial monopole problems. Phys. Lett. B 108, 389 (1982)

8. A.D. Linde, A new inflationary universe scenario: a possible solution of the horizon, flatness, homogeneity, isotropy and primordial monopole problems. Adv. Ser. Astrophys. Cosmol. 3, 149 (1987). https://doi.org/10.1016/0370-2693(82)91219-9

9. A. Albrecht, P.J. Steinhardt, Cosmology for grand unified theories with radiatively induced symmetry breaking. Phys. Rev. Lett. 48, 1220 (1982)

10. A. Albrecht, P.J. Steinhardt, Cosmology for grand unified theories with radiatively induced symmetry breaking. Adv. Ser. Astrophys. Cosmol. 3, 158 (1987). https://doi.org/10.1103/PhysRevLett.48. 1220

11. V. Mukhanov, G. Chibisov, Quantum fluctuation and nonsingular universe (in Russian). JETP Lett. 33, 532 (1981)

12. V. Mukhanov, G. Chibisov, Quantum fluctuation and nonsingular universe (in Russian). Pisma Zh. Eksp. Teor. Fiz. 33, 549 (1981)

13. J.E. Lidsey, A.R. Liddle, E.W. Kolb, E.J. Copeland, T. Barreiro, M. Abney, Reconstructing the inflation potential: an overview. Rev. Mod. Phys. 69, 373 (1997). https://doi.org/10.1103/RevModPhys. 69.37y3. arXiv:astro-ph/9508078

14. R.H. Brandenberger, C. Vafa, Superstrings in the early universe. Nucl. Phys. B 316, 391 (1989)

15. T.D. Brennan, F. Carta, C. Vafa, The string landscape, the swampland, and the missing corner. PoS TASI 2017, 015 (2017). https:// doi.org/10.22323/1.305.0015. arXiv:1711.00864 [hep-th]

16. E. Palti, The swampland: introduction and review. Fortsch. Phys. 67(6), 1900037 (2019). https://doi.org/10.1002/prop.201900037. arXiv:1903.06239 [hep-th]

17. M. van Beest, J. Calderon-Infante, D. Mirfendereski, I. Valenzuela, Lectures on the swampland program in string compactifications. arXiv:2102.01111 [hep-th]

18. H. Ooguri, C. Vafa, On the geometry of the string landscape and the swampland. Nucl. Phys. B 766, 21 (2007)

19. G. Obied, H. Ooguri, L. Spodyneiko, C. Vafa, De Sitter space and the swampland. arXiv:1806.08362 [hep-th]

20. A. Bedroya, C. Vafa, Trans-Planckian censorship and the swampland. arXiv:1909.11063 [hep-th]

21. N. Weiss, Constraints on Hamiltonian lattice formulations of field theories in an expanding universe. Phys. Rev. D 32, 3228 (1985). https://doi.org/10.1103/PhysRevD.32.3228

22. R. Penrose, Gravitational collapse: the role of general relativity. Riv. Nuovo Cim. 1, 252 (1969)

23. R. Penrose, Gravitational collapse: the role of general relativity. Gen. Relativ. Gravit. 34, 1141 (2002)

24. R. Brandenberger, Fundamental physics, the swampland of effective field theory and early universe cosmology. arXiv:1911.06058 [hep-th]

25. P. Agrawal, G. Obied, P.J. Steinhardt, C. Vafa, On the cosmological implications of the string swampland. Phys. Lett. B 784, 271 (2018). https://doi.org/10.1016/j.physletb.2018.07.040. arXiv:1806.09718 [hep-th]

26. L. Heisenberg, M. Bartelmann, R. Brandenberger, A. Refregier, Dark energy in the swampland. Phys. Rev. D 98(12), 123502 (2018). https://doi.org/10.1103/PhysRevD.98.123502. arXiv:1808.02877 [astro-ph.CO]

27. A. Bedroya, R. Brandenberger, M. Loverde, C. Vafa, TransPlanckian censorship and inflationary cosmology. Phys. Rev. D 101(10), 103502 (2020). https://doi.org/10.1103/PhysRevD.101. 103502. arXiv:1909.11106 [hep-th]
28. R. Brandenberger, E. Wilson-Ewing, Strengthening the TCC bound on inflationary cosmology. JCAP 2003(03), 047 (2020). https://doi. org/10.1088/1475-7516/2020/03/047. arXiv:2001.00043 [hep-th]

29. Y. Cai, Y.S. Piao, Pre-inflation and trans-Planckian censorship. Sci. China Phys. Mech. Astron. 63(11), 110411 (2020). https://doi.org/ 10.1007/s11433-020-1573-5. arXiv:1909.12719 [gr-qc]

30. S. Mizuno, S. Mukohyama, S. Pi, Y.L. Zhang, Universal upper bound on the inflationary energy scale from the trans-Planckian censorship conjecture. Phys. Rev. D 102(2), 021301 (2020). https://doi.org/10.1103/PhysRevD.102.021301. arXiv:1910.02979 [astro-ph.CO]

31. M. Dhuria, G. Goswami, Trans-Planckian censorship conjecture and non-thermal post-inflationary history. Phys. Rev. D 100(12), 123518 (2019). https://doi.org/10.1103/PhysRevD.100. 123518. arXiv:1910.06233 [astro-ph.CO]

32. M. Torabian, Non-standard cosmological models and the trans-Planckian censorship conjecture. Fortsch. Phys. 68(2), 1900092 (2020). https://doi.org/10.1002/prop.201900092. arXiv: 1910.06867 [hep-th]

33. H.H. Li, G. Ye, Y. Cai, Y.S. Piao, Trans-Planckian censorship of multi-stage inflation and dark energy. Phys. Rev. D 101(6), 063527 (2020). https://doi.org/10.1103/PhysRevD.101. 063527. arXiv: 1911.06148 [gr-qc]

34. V. Kamali, R. Brandenberger, Relaxing the TCC bound on inflationary cosmology? Eur. Phys. J. C 80(4), 339 (2020). https://doi. org/10.1140/epjc/s10052-020-7908-8. arXiv:2001.00040 [hep-th]

35. G. Dvali, C. Gomez, S. Zell, Quantum break-time of de Sitter. JCAP 1706, 028 (2017). https://doi.org/10.1088/1475-7516/2017/ 06/028. arXiv:1701.08776 [hep-th]

36. G. Dvali, C. Gomez, On exclusion of positive cosmological constant. Fortsch. Phys. 67(1-2), 1800092 (2019). https://doi.org/10. 1002/prop.201800092. arXiv:1806.10877 [hep-th]

37. G. Dvali, C. Gomez, S. Zell, Quantum breaking bound on de Sitter and swampland. Fortsch. Phys. 67(1-2), 1800094 (2019). https:// doi.org/10.1002/prop.201800094. arXiv:1810.11002 [hep-th]

38. S. Brahma, K. Dasgupta, R. Tatar, Four-dimensional de Sitter space is a Glauber-Sudarshan state in string theory. arXiv:2007.00786 [hep-th]

39. S. Brahma, K. Dasgupta, R. Tatar, de Sitter Space as a GlauberSudarshan state. arXiv:2007.11611 [hep-th]

40. H. Bernardo, S. Brahma, K. Dasgupta, R. Tatar, Crisis on infinite earths: short-lived de Sitter vacua in the string theory landscape. arXiv:2009.04504 [hep-th]

41. A. Achucarro, G.A. Palma, The string swampland constraints require multi-field inflation. JCAP 1902, 041 (2019). https://doi. org/10.1088/1475-7516/2019/02/041. arXiv:1807.04390 [hep-th]

42. A. Berera, Warm inflation. Phys. Rev. Lett. 75, 3218 (1995). https:// doi.org/10.1103/PhysRevLett.75.3218. arXiv:astro-ph/9509049

43. S. Das, Warm inflation in the light of swampland criteria. Phys. Rev. D 99(6), 063514 (2019). https://doi.org/10.1103/PhysRevD. 99.063514. arXiv: 1810.05038 [hep-th]

44. M. Motaharfar, V. Kamali, R.O. Ramos, Warm inflation as a way out of the swampland. Phys. Rev. D 99(6), 063513 (2019). https:// doi.org/10.1103/PhysRevD.99.063513. arXiv:1810.02816 [astroph.CO]

45. A. Berera, J.R. Calderón, Trans-Planckian censorship and other swampland bothers addressed in warm inflation. Phys. Rev. D 100(12), 123530 (2019). https://doi.org/10.1103/PhysRevD.100. 123530. arXiv:1910.10516 [hep-ph]

46. S. Das, Distance, de Sitter and trans-Planckian censorship conjectures: the status quo of warm inflation. Phys. Dark Univ. 27, 100432 (2020). https://doi.org/10.1016/j.dark.2019.100432. arXiv:1910.02147 [hep-th] 
47. D.H. Lyth, E.D. Stewart, Thermal inflation and the moduli problem. Phys. Rev. D 53, 1784 (1996). https://doi.org/10.1103/PhysRevD. 53.1784. arXiv:hep-ph/9510204

48. D. Green, B. Horn, L. Senatore, E. Silverstein, Trapped inflation. Phys. Rev. D 80, 063533 (2009). https://doi.org/10.1103/ PhysRevD.80.063533. arXiv:0902.1006 [hep-th]

49. P. Adshead, M. Wyman, Chromo-natural inflation: natural inflation on a steep potential with classical non-Abelian gauge fields. Phys. Rev. Lett. 108, 261302 (2012). https://doi.org/10.1103/ PhysRevLett.108.261302. arXiv:1202.2366 [hep-th]

50. V. Kamali, Reheating after swampland conjecture. JHEP 01, 092 (2020). https://doi.org/10.1007/JHEP01(2020)092. arXiv: 1902.00701 [gr-qc]

51. S. Laliberte, R. Brandenberger, String gases and the swampland. JCAP 2007, 046 (2020). https://doi.org/10.1088/1475-7516/2020/ 07/046. arXiv:1911.00199 [hep-th]

52. G.A. Mitchell, R. Brandenberger, Note on shape moduli stabilization, string gas cosmology and the swampland criteria. Eur. Phys. J. C 81(1), 39 (2021). https://doi.org/10.1140/epjc/ s10052-021-08843-0. arXiv:2008.13251 [hep-th]

53. H. Ooguri, E. Palti, G. Shiu, C. Vafa, Distance and de Sitter conjectures on the swampland. Phys. Lett. B 788, 180 (2019). https://doi. org/10.1016/j.physletb.2018.11.018. arXiv:1810.05506 [hep-th]

54. S.K. Garg, C. Krishnan, Bounds on slow roll and the de Sitter swampland. JHEP 1911, 075 (2019). https://doi.org/10.1007/ JHEP11(2019)075. arXiv:1807.05193 [hep-th]

55. D. Baumann, L. McAllister, Inflation and string theory. https://doi. org/10.1017/CBO9781316105733. arXiv:1404.2601 [hep-th]

56. M.P. Hertzberg, S. Kachru, W. Taylor, M. Tegmark, Inflationary constraints on type IIA string theory. JHEP 0712, 095 (2007). https://doi.org/10.1088/1126-6708/2007/12/095. arXiv:0711.2512 [hep-th]

57. D. Kutasov, T. Maxfield, I. Melnikov, S. Sethi, Constraining de Sitter space in string theory. Phys. Rev. Lett. 115(7), 071305 (2015). https://doi.org/10.1103/PhysRevLett.115.071305. arXiv:1504.00056 [hep-th]

58. S. Sethi, Supersymmetry breaking by fluxes. JHEP 1810, 022 (2018). https://doi.org/10.1007/JHEP10(2018)022. arXiv:1709.03554 [hep-th]

59. K. Dasgupta, M. Emelin, E. McDonough, R. Tatar, Quantum corrections and the de Sitter swampland conjecture. JHEP 1901, 145 (2019). https://doi.org/10.1007/JHEP01(2019)145. arXiv:1808.07498 [hep-th]

60. K. Dasgupta, M. Emelin, M.M. Faruk, R. Tatar, de Sitter vacua in the string landscape. arXiv:1908.05288 [hep-th]

61. M. Scalisi, I. Valenzuela, Swampland distance conjecture, inflation and $\alpha$-attractors. JHEP 1908, 160 (2019). https://doi.org/10.1007/ JHEP08(2019)160. arXiv:1812.07558 [hep-th]

62. R.H. Brandenberger, J.H. Kung, Chaotic inflation as an attractor in initial condition space. Phys. Rev. D 42, 1008 (1990). https://doi. org/10.1103/PhysRevD.42.1008

63. R. Brandenberger, Initial conditions for inflation-a short review. Int. J. Mod. Phys. D 26(01), 1740002 (2016). https://doi.org/10. 1142/S0218271817400028. arXiv:1601.01918 [hep-th]

64. D.S. Goldwirth, T. Piran, Initial conditions for inflation. Phys. Rep. 214, 223 (1992). https://doi.org/10.1016/0370-1573(92)90073-9

65. A. Berera, S. Brahma, J.R. Calderón, Role of trans-Planckian modes in cosmology. JHEP 08, 071 (2020). https://doi.org/10. 1007/JHEP08(2020)071. arXiv:2003.07184 [hep-th]

66. G. Dvali, A. Kehagias, A. Riotto, Inflation and decoupling. arXiv:2005.05146 [hep-th]

67. C.P. Burgess, S.P. de Alwis, F. Quevedo, Cosmological transPlanckian conjectures are not effective. arXiv:2011.03069 [hep-th]

68. L. Dolan, R. Jackiw, Symmetry behavior at finite temperature. Phys. Rev. D 9, 3320 (1974). https://doi.org/10.1103/PhysRevD. 9.3320
69. S. Watson, Moduli stabilization with the string Higgs effect. Phys. Rev. D 70, 066005 (2004). https://doi.org/10.1103/PhysRevD.70. 066005. arXiv:hep-th/0404177

70. L. Kofman, A.D. Linde, X. Liu, A. Maloney, L. McAllister, E. Silverstein, Beauty is attractive: moduli trapping at enhanced symmetry points. JHEP 0405, 030 (2004). https://doi.org/10.1088/ 1126-6708/2004/05/030. arXiv:hep-th/0403001

71. D.J.H. Chung, E.W. Kolb, A. Riotto, I.I. Tkachev, Probing Planckian physics: resonant production of particles during inflation and features in the primordial power spectrum. Phys. Rev. D 62, 043508 (2000). https://doi.org/10.1103/PhysRevD.62.043508. arXiv:hep-ph/9910437

72. A.D. Dolgov, D.P. Kirilova, On particle creation by a time dependent scalar field. Sov. J. Nucl. Phys. 51, 172 (1990)

73. A.D. Dolgov, D.P. Kirilova, On particle creation by a time dependent scalar field. Yad. Fiz. 51, 273 (1990)

74. J.H. Traschen, R.H. Brandenberger, Particle production during outof-equilibrium phase transitions. Phys. Rev. D 42, 2491 (1990). https://doi.org/10.1103/PhysRevD.42.2491

75. L. Kofman, A.D. Linde, A.A. Starobinsky, Reheating after inflation. Phys. Rev. Lett. 73, 3195 (1994). https://doi.org/10.1103/ PhysRevLett.73.3195. arXiv:hep-th/9405187

76. Y. Shtanov, J.H. Traschen, R.H. Brandenberger, Universe reheating after inflation. Phys. Rev. D 51, 5438 (1995). https://doi.org/10. 1103/PhysRevD.51.5438. arXiv:hep-ph/9407247

77. L. Kofman, A.D. Linde, A.A. Starobinsky, Towards the theory of reheating after inflation. Phys. Rev. D 56, 3258 (1997). https://doi. org/10.1103/PhysRevD.56.3258. arXiv:hep-ph/9704452

78. R. Allahverdi, R. Brandenberger, F.Y. Cyr-Racine, A. Mazumdar, Reheating in inflationary cosmology: theory and applications. Annu. Rev. Nucl. Part. Sci. 60, 27 (2010). https://doi.org/10.1146/ annurev.nucl.012809.104511. arXiv:1001.2600 [hep-th]

79. M.A. Amin, M.P. Hertzberg, D.I. Kaiser, J. Karouby, Nonperturbative dynamics of reheating after inflation: a review. Int. J. Mod. Phys. D 24, 1530003 (2014). https://doi.org/10.1142/ S0218271815300037. arXiv:1410.3808 [hep-ph]

80. A. Berera, M. Gleiser, R.O. Ramos, A first principles warm inflation model that solves the cosmological horizon/flatness problems. Phys. Rev. Lett. 83, 264-267 (1999). https://doi.org/10.1103/ PhysRevLett.83.264. arXiv:hep-ph/9809583 [hep-ph]

81. A. Berera, T.W. Kephart, The ubiquitous inflaton in string-inspired models. Phys. Rev. Lett. 83, 1084-1087 (1999). https://doi.org/10. 1103/PhysRevLett.83.1084. arXiv:hep-ph/9904410

82. K. Freese, J.A. Frieman, A.V. Olinto, Natural inflation with pseudoNambu-Goldstone bosons. Phys. Rev. Lett. 65, 3233 (1990). https://doi.org/10.1103/PhysRevLett.65.3233

83. A. Maleknejad, M.M. Sheikh-Jabbari, Gauge-flation: inflation from non-Abelian gauge fields. Phys. Lett. B 723, 224 (2013). https://doi.org/10.1016/j.physletb.2013.05.001. arXiv:1102.1513 [hep-ph]

84. A. Maleknejad, M.M. Sheikh-Jabbari, Non-Abelian gauge field inflation. Phys. Rev. D 84, 043515 (2011). https://doi.org/10.1103/ PhysRevD.84.043515. arXiv:1102.1932 [hep-ph]

85. A. Maleknejad, M.M. Sheikh-Jabbari, J. Soda, Gauge fields and inflation. Phys. Rep. 528, 161 (2013). https://doi.org/10.1016/j. physrep.2013.03.003. arXiv:1212.2921 [hep-th]

86. M. Dine, J.A.P. Law-Smith, S. Sun, D. Wood, Y. Yu, Obstacles to constructing de Sitter space in string theory. JHEP 02, 050 (2021). https://doi.org/10.1007/JHEP02(2021)050. arXiv:2008.12399 [hep-th]

87. J. Martin, C. Ringeval, R. Trotta, V. Vennin, The best inflationary models after planck. JCAP 1403, 039 (2014). https://doi.org/10. 1088/1475-7516/2014/03/039. arXiv:1312.3529 [astro-ph.CO] 
88. S. Bartrum, M. Bastero-Gil, A. Berera, R. Cerezo, R.O. Ramos, J.G. Rosa, The importance of being warm (during inflation). Phys. Lett. B 732, 116-121 (2014). https://doi.org/10.1016/j.physletb. 2014.03.029. arXiv:1307.5868 [hep-ph]
89. M. Bastero-Gil, A. Berera, R.O. Ramos, J.G. Rosa, Warm little inflaton. Phys. Rev. Lett. 117(15), 151301 (2016). https://doi.org/ 10.1103/PhysRevLett.117.151301. arXiv:1604.08838 [hep-ph] 